Author affiliations and support information (if applicable) appear at the end of this article.

Published at jco.org on June 28, 2018. F.C. and E.B. shared first authorship. M.v.d.L. and A.S. shared last authorship. Clinical trial information: NCT02138513. Corresponding author: Félix Compen, MSc, Radboud universitair medisch centrum voor mindfulness, Postbus 9101 6500 HB Nijmegen, the Netherlands; e-mail: felixcompen@radboudumc.nl.

(C) 2018 by American Society of Clinical Oncology

$0732-183 X / 18 / 3623 w-2413 w / \$ 20.00$

\title{
Face-to-Face and Internet-Based Mindfulness-Based Cognitive Therapy Compared With Treatment as Usual in Reducing Psychological Distress in Patients With Cancer: A Multicenter Randomized Controlled Trial
}

Félix Compen, Else Bisseling, Melanie Schellekens, Rogier Donders, Linda Carlson, Marije van der Lee, and Anne Speckens

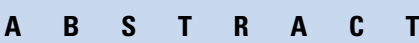

\section{Purpose}

Mindfulness-based cognitive therapy (MBCT) has been shown to alleviate psychological distress in patients with cancer. However, patients experience barriers to participating in face-to-face MBCT. Individual Internet-based MBCT (eMBCT) could be an alternative. The study aim was to compare MBCT and EMBCT with treatment as usual (TAU) for psychological distress in patients with cancer.

\section{Patients and Methods}

We obtained ethical and safety approval to include 245 patients with cancer with psychological distress ( $\geq 11$ on the Hospital Anxiety and Depression Scale) in the study. They were randomly allocated to MBCT ( $n=77$ ), eMBCT $(n=90)$, or TAU $(n=78)$. Patients completed baseline (T0) and postintervention (T1) assessments. The primary outcome was psychological distress on the Hospital Anxiety and Depression Scale. Secondary outcomes were psychiatric diagnosis, fear of cancer recurrence, rumination, health-related quality of life, mindfulness skills, and positive mental health. Continuous outcomes were analyzed using linear mixed modeling on the intention-to-treat sample. Because both interventions were compared with TAU, the type I error rate was set at $P<.025$.

\section{Results}

Compared with TAU, patients reported significantly less psychological distress after both MBCT (Cohen's d, .45; $P<.001$ ) and eMBCT (Cohen's d, .71; $P<.001$ ). In addition, post-treatment prevalence of psychiatric diagnosis was lower with both $\mathrm{MBCT}(33 \%$ improvement; $P=.030)$ and eMBCT (29\% improvement; $P=.076)$ in comparison with TAU $(16 \%)$, but these changes were not statistically significant. Both interventions reduced fear of cancer recurrence and rumination, and increased mental health-related quality of life, mindfulness skills, and positive mental health compared with TAU (all PS $<$.025). Physical health-related quality of life did not improve $(P=.343)$.

\section{Conclusion}

Compared with TAU, MBCT and eMBCT were similarly effective in reducing psychological distress in a sample of distressed heterogeneous patients with cancer.

\section{J Clin Oncol 36:2413-2421. (C) 2018 by American Society of Clinical Oncology}

\section{INTRODUCTION}

From 2025 onward, 20 million people worldwide will be diagnosed with cancer each year. ${ }^{1}$ Approximately one third of patients with cancer suffer from significant psychological distress, ${ }^{2}$ resulting in reduced quality of life, decreased compliance with medical care, and prolonged duration of hospital stay. ${ }^{3,4}$ The prevalence of psychiatric disorders in oncologic settings is 30\% to $40 \%$. $^{3}$ Effective and accessible interventions are needed to reduce psychological distress and psychiatric disorders in patients with cancer.

Mindfulness-based interventions (MBIs) $)^{5,6}$ such as mindfulness-based cognitive therapy (MBCT), teach participants to be more mindful in daily life through meditation exercises, yoga, group discussions, and didactic teaching. ${ }^{5}$ A 2012 meta-analysis of randomized controlled trials (RCTs) of MBIs in 955 patients with cancer found significant improvements in depressive and anxiety symptoms. ${ }^{7}$ Since then, a number of RCTs have confirmed this. ${ }^{8-13}$ 
However, because MBIs typically require in-person attendance at classes over several weeks, many patients with cancer experience barriers to participation. These may include impairments due to illness and anticancer treatments, adverse effects that result in advice to avoid groups of people, or limited transportation options. ${ }^{14}$ Consequently, uptake of face-to-face interventions for patients with cancer has been lower than, for instance, telephonebased interventions. ${ }^{15}$

In contrast, Internet-based interventions are easily accessible and save traveling time. ${ }^{16}$ Therapist-guided Internet interventions have been shown to be effective for psychiatric and somatic conditions. ${ }^{17}$ Although evidence for Internet-based MBIs (eMBIs) in cancer is scarce, one controlled study of 62 patients found that synchronous videoconferencing sessions led to significant improvements in mood, stress symptoms, and mindfulness skills, ${ }^{18}$ In addition, an uncontrolled cohort of 257 fatigued patients showed significant improvements in fatigue and psychological distress after individual eMBCT. ${ }^{19}$

To date, no study has simultaneously compared the effectiveness of both MBCT and Internet-based MBCT (eMBCT) with treatment as usual (TAU). The primary aim of this RCT was to investigate whether MBCT and eMBCT were each superior to TAU in reducing psychological distress in a sample of distressed patients with cancer. Moreover, we hypothesized that there would be a reduction of psychiatric diagnoses, fear of cancer recurrence, and rumination, and an improvement in health-related quality of life, mindfulness skills, and positive mental health in both interventions compared with TAU. We explored moderators of intervention dropout and outcome in the interventions: sex, age, cancer diagnosis, anticancer treatment intent, psychiatric diagnosis, neuroticism, and therapist.

\section{PATIENTS AND METHODS}

\section{Trial Design}

A three-armed multicenter, parallel group RCT was conducted to compare the effectiveness of MBCT and eMBCT with TAU in reducing psychological distress in patients with cancer. Anticipated dropout rates were $15 \%$ in MBCT and TAU, and $30 \%$ in eMBCT. ${ }^{19}$ Given the anticipated dropout rates, the allocation ratio was 1:1.2:1. Patients randomly assigned to receive TAU were secondarily randomly assigned to MBCT or eMBCT, to be given after the TAU period of 3 months. The study was approved by the ethical review board of the Radboud University Medical Center (CMO Arnhem-Nijmegen 2013/542). All centers provided local ethics approval. The study was registered on Clinicaltrials.gov (NCT02138513) shortly after the start of recruitment and was reported following CONSORT guidelines. ${ }^{20}$ A protocol article was published in advance of trial completion. ${ }^{21}$

\section{Participants}

Inclusion criteria were (1) a cancer diagnosis, any tumor type or stage, at any time, receiving or not receiving treatment; (2) a score of $\geq 11$ on the Hospital Anxiety and Depression Scale (HADS); (3) computer literacy and Internet access; (4) ability to participate in both MBCT and eMBCT; and (5) good command of the Dutch language. Exclusion criteria were (1) severe psychiatric morbidity, such as suicidal ideation and/or current psychosis; (2) change in psychotropic medication within a period of 3 months before baseline; and (3) previous participation in four or more sessions of an MBI.

\section{Procedure}

Patients were recruited from April 2014 to December 2015 via health care professionals in six centers $(n=64 ; 26 \%)$ via online media $(n=49$; $20 \%)$, offline media $(n=44 ; 18 \%)$, patient associations $(n=43 ; 18 \%)$, and peers $(n=27 ; 11 \%)$. Eighteen patients $(7 \%)$ could not remember how they heard about the study. Interested patients filled out the HADS on the research Web site. Patients with HADS $\geq 11$ received a phone call from one of the researchers, during which the remaining inclusion and exclusion criteria were assessed. Once patients provided oral and written consent and had completed the baseline assessment, they were randomly assigned to MBCT, eMBCT, or TAU and informed about their allocation by E.B.

\section{Intervention}

Face-to-face MBCT. The MBCT protocol ${ }^{6}$ was tailored to patients with cancer by including cancer-related psychoeducation and adapted movement exercises. The MBCT consisted of eight weekly 2.5-hour group sessions, a 6-hour silent day, and daily home practice assignments guided by audio files. Each participant in both interventions received a folder with information on each session.

Internet-based mindfulness-based cognitive therapy. The eMBCT was delivered individually and included weekly asynchronous written interaction with a therapist over e-mail. Patients were granted access to a secure Web site containing material for 8 weeks plus a silent day and an inbox. Each session included an introduction and daily meditation exercises with meditation audio files. Patients were asked to practice and fill out practice diaries on a daily basis. They were provided with (fictional) patients' descriptions to emphasize common experiences and clarify the use of the diaries. Patients were given written instructions after week 5 to prepare for their silent day at home. In the week of the silent day, patients were provided with a program similar to the MBCT silent day. At the end of the silent day, eMBCT patients wrote about their experiences in an essay. The therapist provided written feedback on the completed forms and the essay via the secured inbox on a prearranged day of the week. Having completed four or more sessions of MBCT was defined as a minimum adequate dose in both interventions. ${ }^{22}$

Treatment as usual. TAU consisted of all health care that patients usually received. Except for not participating in MBIs during the study period, there were no restrictions on health care utilization. Data on health care utilization were gathered using the Trimbos/iMTA questionnaire for Costs associated with Psychiatric illnesses. ${ }^{23}$

\section{Therapists}

Fourteen therapists participated: seven provided both interventions, two only provided MBCT, and five only provided eMBCT. All therapists fulfilled the criteria of the UK Mindfulness-Based Teacher Therapist Network Good Practice Guidelines for teaching MBIs. ${ }^{24}$ Three full-day supervision meetings were held during the intervention phase of the trial. All face-to-face MBCT sessions were videotaped to evaluate therapist competency using the Mindfulness-Based Interventions-Teachers Assessment Criteria. ${ }^{25}$ Therapist competency levels were determined by two independent therapists who evaluated two random sessions from each of the nine therapists providing face-to-face MBCT (who treated $80.8 \%$ of all patients receiving either intervention). Interrater reliability was .72 . Of the nine therapists rated, four were considered proficient ( $n=64$ patients), three were considered competent ( $\mathrm{n}=64$ patients), and two were considered beginner ( $n=7$ patients).

\section{Measures}

Primary outcome. Psychological distress was measured with the HADS, a 14-item self-report scale designed to assess anxiety and depression in medical outpatients. ${ }^{26,27}$ It has good psychometric properties in the general medical population, including patients with cancer in palliative care. $^{28}$ The internal consistency in this sample was high $(\alpha=.87)$. 
Secondary outcomes. Psychiatric diagnosis was assessed by the Structured Clinical Interview for DSM-IV-TR Axis I Disorders. ${ }^{29}$ The Structured Clinical Interview for DSM-IV-TR Axis I Disorders was administered by a trained interviewer who completed a Master in Behavioral Science degree (F.C.), supervised by either an experienced psychiatrist (E.B. or A.S.) or psychologist (M.v.d.L.). All interviews were audiotaped. Fear of cancer recurrence was assessed with the severity subscale of the Fear of Cancer Recurrence Inventory. ${ }^{30,31}$ Rumination was measured by the rumination subscale of the Rumination and Reflection Questionnaire; ${ }^{32}$ health-related quality of life was measured by the mental and physical scales of the Short-Form $12^{33}$ using Dutch norm scores from a clinical sample ${ }^{34}$ mindfulness skills were measured by the Five Facet Mindfulness Questionnaire-Short Form; ${ }^{35}$ and positive mental health was measured by the Mental Health Continuum-Short Form. ${ }^{36}$ As a potential moderator, neuroticism was measured by the Neuroticism Extraversion Openness-Five Factor Inventory. ${ }^{37}$ Further details of the measures used are included in the study protocol (Data Supplement). ${ }^{21}$

\section{Sample Size}

The sample size calculation was based on previous postintervention HADS scores of patients with cancer who received MBCT at the Helen Dowling Institute (mean, 10.6; standard deviation [SD], 6.4) compared with those who had not received it (mean, 14.8; SD, 8.1). With $90 \%$ power, 65 patients per condition were needed. Because of anticipated differential dropout rates among treatment arms, the recruitment target was 245 patients: 76 in each of the MBCT and TAU arms, and 93 in the eMBCT arm.

\section{Randomization and Blinding}

Once patients provided oral and written consent and completed the baseline assessment, they were randomly assigned to MBCT, eMBCT, or TAU by a computer-generated allocation sequence designed by an independent biostatistician. This custom software was accessed by one of the researchers (E.B.) via a study-specific Web site. Randomization was carried out with a fixed block size of 16 stratified for region and minimized for sex, cancer diagnosis (breast $v$ other), and anticancer treatment intent (curative $v$ palliative). After randomization, E.B. informed patients of their allocation by e-mail. E.B. planned and invited participants to the follow-up assessments; the standardized psychiatric interviews were conducted by F.C. and research assistants who were blinded to treatment allocation. Both E.B. and F.C. instructed patients not to mention their treatment condition at the beginning of each psychiatric interview.

\section{Statistical Analysis}

Statistical analyses were carried out using Statistical Package for the Social Sciences (SPSS) version 22 (SPSS, Chicago, IL). Differences among conditions in demographic and clinical variables were tested by $\chi^{2}$ analysis and $t$ tests. Continuous outcomes were analyzed with linear mixed modeling in a model with uncorrelated residual errors and random intercepts, including group allocation and its interaction with time and stratification (region) and minimization (sex, cancer diagnosis, anticancer treatment intent) variables as fixed factors. Because both MBCT and eMBCT were compared with TAU, the two-sided type I error rate was corrected to .025 for the two direct (MBCT and eMBCT) comparisons with TAU. All reported analyses used the intent-to-treat sample. Missing continuous outcomes were imputed with automatic multiple imputation on the basis of linear regression (20 iterations). The multiple imputation data set was considered the primary data set. Cohen's d effect sizes were calculated using postintervention means and baseline pooled SDs. These statistics are commonly used in psychological contexts to compare effect sizes across studies, ${ }^{38}$ and in accordance with Cohen's guidelines, Cohens's d effect sizes were interpreted as small ( 0.2 to 0.5$)$, medium ( 0.5 to 0.8$)$, or large $(>0.8) .{ }^{39}$

In addition, the reliable change index (RCI) was calculated by dividing the observed difference score by the SE of measurement. Each participant was categorized as improved $(\mathrm{RCI}<-1.96)$, no change $(-1.96$ to 1.96 ), or deteriorated ( $\mathrm{RCI}>1.96) .{ }^{40}$ Improvements in terms of psychiatric diagnosis and RCI were assessed using $\chi^{2}$ analyses.

Exploratory moderation analyses of dropout were performed using logistic regression, including an interaction term between completer (yes/ no) and possible moderators: sex, age, cancer diagnosis, anticancer treatment intent, psychiatric diagnosis, neuroticism, and therapist. Exploratory moderation analyses of the primary outcome were performed by including a three-way interaction term among condition, time, and possible moderators: sex, age, cancer diagnosis, anticancer treatment intent, psychiatric diagnosis, neuroticism, and therapist. First, moderators were assessed in two separate analyses of either intervention compared with TAU. Second, moderators were assessed in analyses of the two intervention conditions only.

\section{RESULTS}

\section{Study Sample}

In total, 532 patients were screened with the HADS (Fig 1), of whom $98(18.4 \%)$ were excluded for scoring $<11$. Of 434 patients who were contacted by telephone, $24(5.5 \%)$ were excluded because of previous experience with mindfulness, 22 (5.1\%) could not be contacted, and 95 (21.9\%) declined participation because of possible traveling distance $(\mathrm{n}=55 ; 12.7 \%)$; strong randomization preference $(\mathrm{n}=12 ; 2.8 \%)$, of whom four had a preference for eMBCT and eight for in-person group MBCT; and scheduling difficulties ( $\mathrm{n}=11 ; 2.5 \%)$. Of the remaining 293 patients, another $10(3.4 \%)$ could not be contacted, and 38 (13.0\%) declined participation after the baseline assessment. There were no significant differences in mean HADS scores between the 133 decliners (mean, 20.4; SD, 5.6) and those who were randomly assigned (mean, 20.6; $\mathrm{SD}, 6.2$ ). In total, 245 patients with cancer were randomly assigned to MBCT $(n=77)$, eMBCT $(n=90)$, or TAU $(n=78)$. The three conditions did not differ in terms of baseline demographic or clinical characteristics (Table 1). The number of months between baseline and postintervention assessments did not differ between MBCT (mean, 5.4; SD, 2.3) and eMBCT (mean, 5.9; SD, 1.8; $P=.13$ ), but was higher in both intervention conditions than in TAU (mean, 3.5; SD, $0.9 ; P<.001)$.

Seventy of 77 patients (90.9\%) started MBCT, and $71(92.2 \%)$ completed four or more sessions (mean, 7.9; SD, 1.3; Fig 1). Eightytwo of 90 patients $(91.1 \%)$ started eMBCT and 71 completed four or more sessions (mean, 8.6; SD, 12). The amount of estimated daily minutes of mindfulness practice did not differ significantly between MBCT ( $\mathrm{n}=56$; mean, 30.6; SD, 26.0) and eMBCT ( $\mathrm{n}=70$; mean, 28.7; SD, 29.3; $P=.69$ ). Dropouts from the interventions were significantly higher in the eMBCT than in the MBCT group: $\chi^{2}(1, n$ $=167)=3.92(P=.047)$. Nonresponse on the post-treatment assessment was substantial (16.9\% in MBCT, $16.7 \%$ in eMBCT, and $10.3 \%$ in TAU) but did not differ significantly among conditions $(P$ $=.41)$. Nonresponders were more often female $(P=.033)$ and had less education $(P=.037)$ than responders.

\section{Health Care Utilization}

There were no significant differences in health care utilization between the intervention and TAU groups (Table 2), except for the proportion of patients receiving outpatient treatment (eg, chemotherapy), which was higher in the TAU group. 


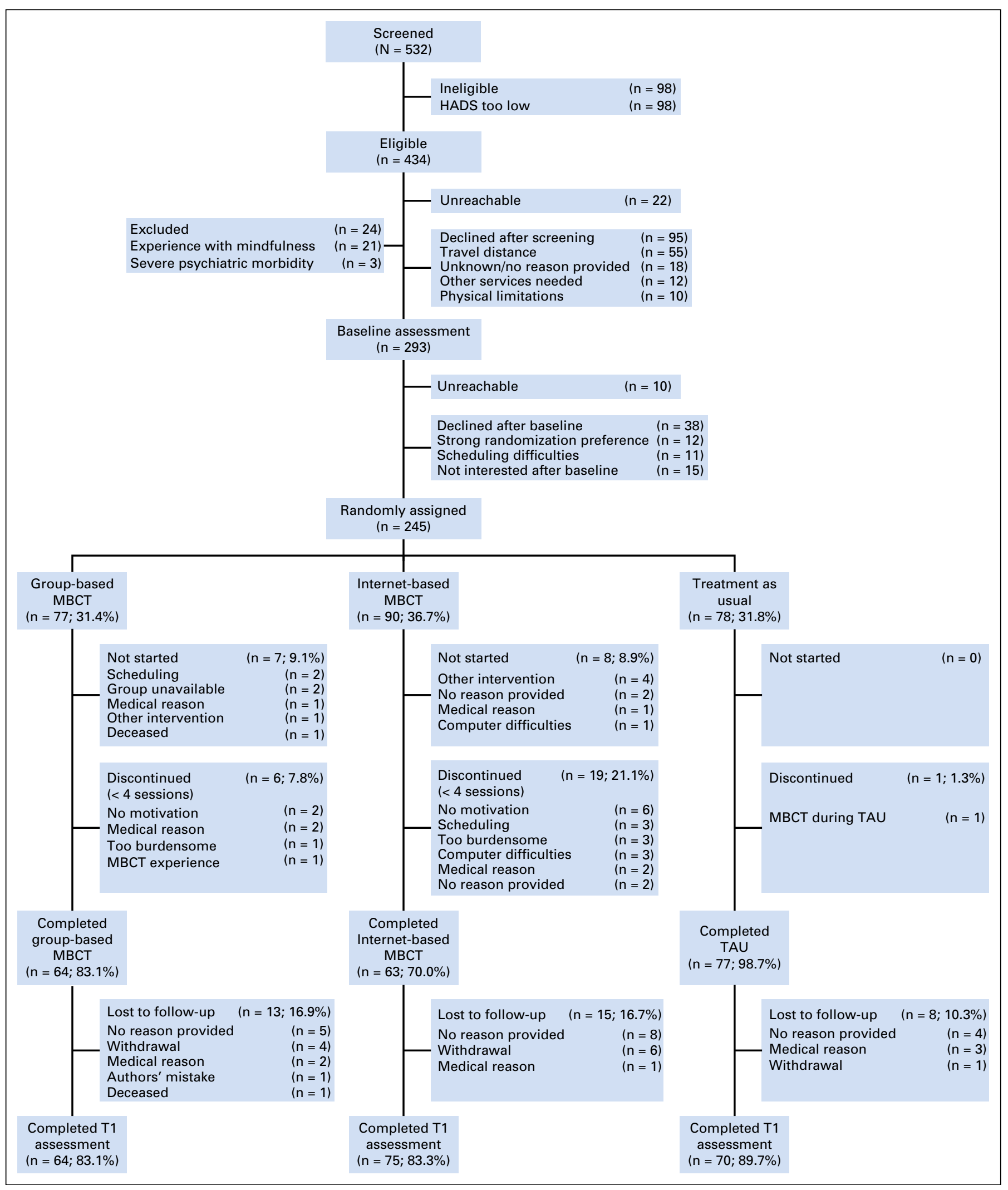

Fig 1. CONSORT diagram ( $n=245$ ). HADS, Hospital Anxiety and Depression Scale; MBCT, mindfulness-based cognitive therapy; TAU, treatment as usual. 


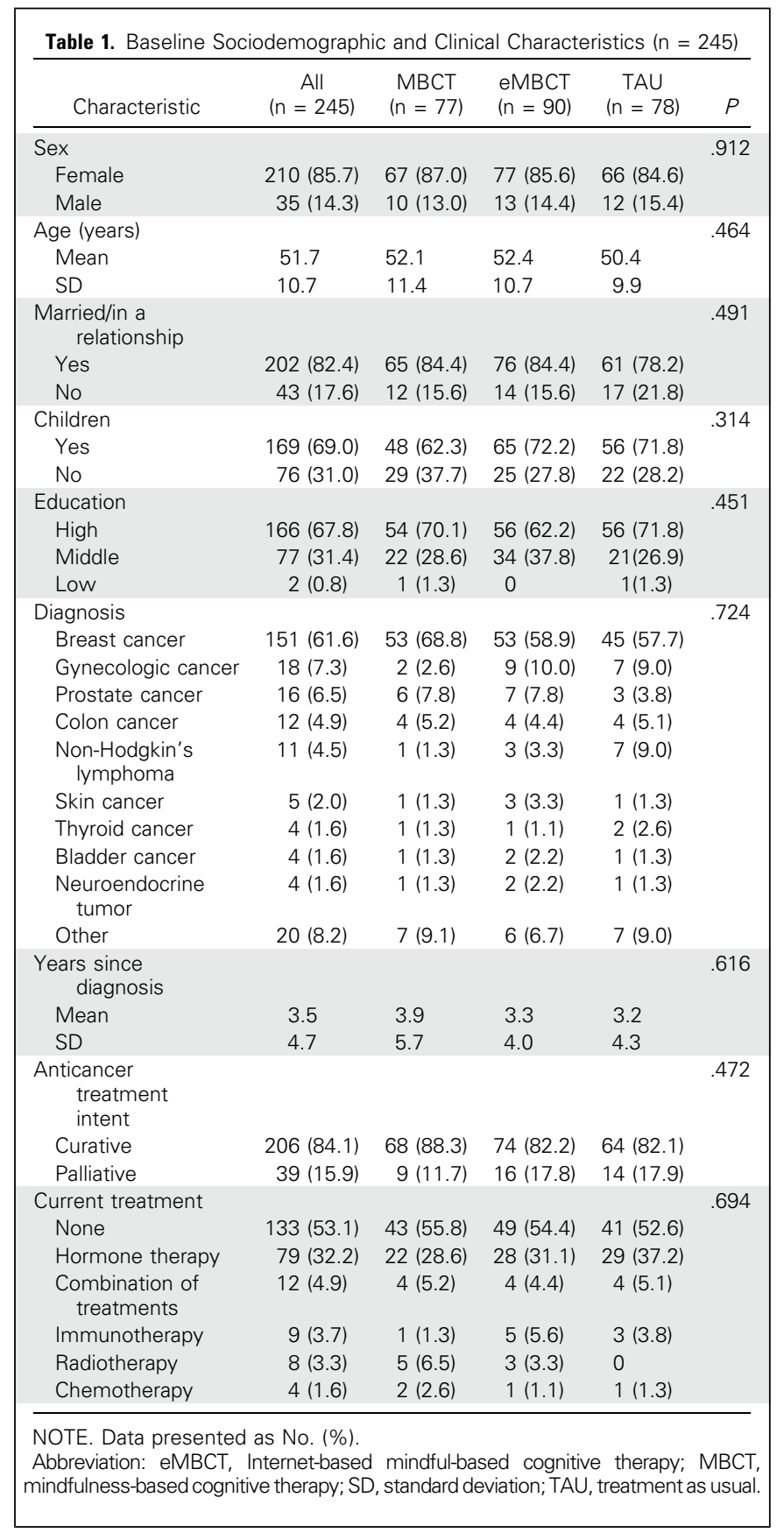

\section{Safety}

A total of 21 severe adverse events unrelated to the intervention were reported in the MBCT $(n=6)$, eMBCT $(n=9)$, and TAU $(n=6)$ groups (Appendix Table A1, online only). One severe adverse event occurred during the study period: a patient died after being randomly assigned as a result of illness.

\section{Intervention Outcomes}

In between-group comparisons of both interventions compared with TAU, patients in the MBCT and eMBCT conditions reported significantly less psychological distress postintervention than did those receiving TAU, with small to medium effect sizes
(Cohen's d, .45 and .71, respectively; Table 3; Fig 2). The proportion of patients demonstrating reliable improvement was significantly greater in MBCT than TAU $\left(36 \% v 14 \% ; \chi^{2}[1, \mathrm{n}=134]=\right.$ $8.44 ; \mathrm{P}=.004)$ and in eMBCT than TAU $\left(37 \% \mathrm{v} 14 \% ; \chi^{2}[1, \mathrm{n}=\right.$ $145]=9.95 ; \mathrm{P}=.002)$. Improvement in rates of psychiatric diagnosis favored both interventions compared with TAU but were not statistically significant (MBCT: $32 \%$ v $16 \% ; \chi^{2}[1, \mathrm{n}=126]=4.73$; $\mathrm{P}=.030$; and eMBCT: $29 \%$ v $\left.16 \% ; \chi^{2}[1, \mathrm{n}=138]=3.15 ; P=.076\right)$.

Compared with TAU, both MBCT and eMBCT significantly reduced fear of cancer recurrence (Cohen's d, .27 and .53, respectively), rumination (Cohen's d, .42 and .51, respectively), and improved mental health-related quality of life (Cohen's d, .59 and .67 , respectively), but not physical health-related quality of life (Cohen's d, .35 and .24 , respectively). They also resulted in better mindfulness skills (Cohen's d, .47 and .82, respectively) and increased positive mental health compared with TAU (Cohen's d, .12 and .44 , respectively).

\section{Moderation}

Exploratory analyses yielded no significant moderation of intervention dropout or primary outcome in the analyses comparing both interventions with TAU separately (all $P$ s $>.05$ ), except for neuroticism. In the analyses comparing either MBCT interventions with TAU, there was a significant interaction between neuroticism and intervention condition (MBCT $v$ TAU $P=.014$; eMBCT $v$ TAU $P=.004)$. Patients scoring higher on neuroticism on baseline improved more on psychological distress in both intervention conditions than in TAU.

\section{DISCUSSION}

To our knowledge, this is the first study to simultaneously compare MBCT and eMBCT with TAU in a large sample of distressed heterogeneous patients with cancer. Both MBCT and eMBCT resulted in a statistically significant and clinically reliable reduction of psychological distress compared with TAU (Table 4). Both interventions demonstrated similar reductions of fear of cancer recurrence, rumination, and improvements in mental (but not physical) health-related quality of life, mindfulness skills, and positive mental health compared with TAU.

Our study confirms previous findings regarding the effectiveness of eMBIs for patients with cancer. ${ }^{14,19}$ Although the group-based setting is considered important for MBIs, ${ }^{41}$ this study suggests that individual guided eMBCT with limited teacher feedback is also effective, thus improving the accessibility of this intervention for patients with cancer. However, eMBCT did result in higher dropout rates than MBCT. Exploratory analyses did not yield any moderators of intervention dropout. Furthermore, qualitative research examining the reasons for dropout is critical to improve the efficacy of Web-based interventions. ${ }^{42}$

A strength of this study is the patient-centered nature of the recruitment across multiple regions because in previous research, we encountered difficulties with consecutive sampling of patients in hospital outpatient settings. ${ }^{43}$ Other strengths are that the interventions followed strict protocols, they were delivered by qualified therapists, and therapist competency was rated by two 


\begin{tabular}{|c|c|c|c|c|c|c|c|c|c|}
\hline \multirow[b]{2}{*}{ Utilization } & \multicolumn{2}{|c|}{ All $(n=198)$} & \multicolumn{2}{|c|}{ MBCT $(n=63)$} & \multicolumn{2}{|c|}{ eMBCT $(n=72)$} & \multicolumn{2}{|c|}{ TAU $(n=63)$} & \multirow[b]{2}{*}{$P^{*}$} \\
\hline & No. & $\%$ & No. & $\%$ & No. & $\%$ & No. & $\%$ & \\
\hline Hospital outpatient consultation & 153 & 77 & 45 & 71 & 61 & 85 & 47 & 75 & .153 \\
\hline Hospital overnight & 13 & 7 & 4 & 9 & 4 & 6 & 5 & 8 & .853 \\
\hline Hospital outpatient treatment & 36 & 18 & 6 & 10 & 12 & 17 & 18 & 29 & .020 \\
\hline Hospital emergency department & 7 & 4 & 1 & 1 & 3 & 3 & 3 & 4 & .588 \\
\hline Mental health care $\dagger$ & 62 & 31 & 18 & 29 & 24 & 33 & 20 & 32 & .834 \\
\hline General practitioner & 116 & 59 & 35 & 56 & 39 & 54 & 42 & 67 & .285 \\
\hline Physical therapist & 92 & 47 & 29 & 46 & 40 & 56 & 23 & 37 & .086 \\
\hline Complementary care & 56 & 28 & 21 & 33 & 20 & 28 & 15 & 34 & .491 \\
\hline
\end{tabular}

independent, experienced therapists. We systematically collected data on health care utilization during the study. The study used a broad array of outcome measures, including both observer-rated interviews and self-report questionnaires.

In addition to these strengths, the study has some limitations. The study was not powered to directly compare the two interventions or determine noninferiority of eMBCT to MBCT, because this would have required a larger sample size. As with other psycho-oncology research, the majority of the participants were middle-aged patients with breast cancer. Although this is in line with the characteristics of patients with cancer seeking psychosocial support, ${ }^{44}$ this might limit generalizability to patients with other

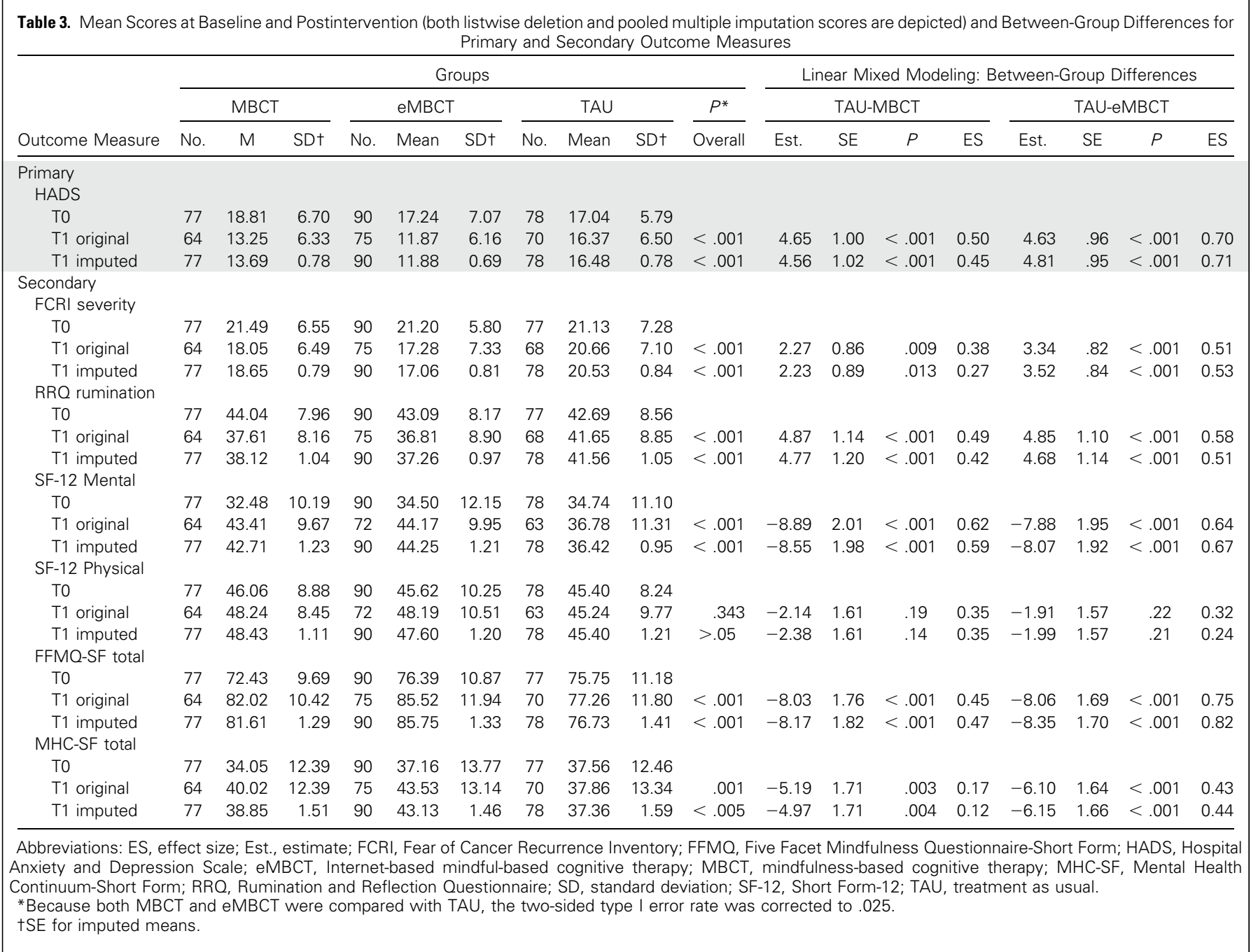




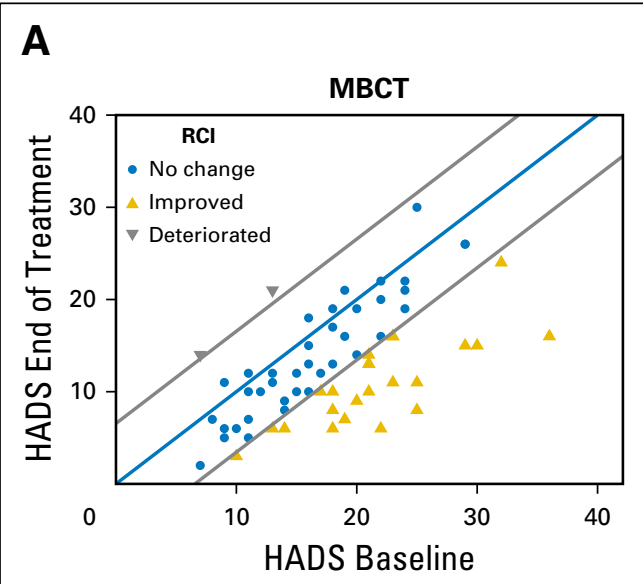

\section{B}

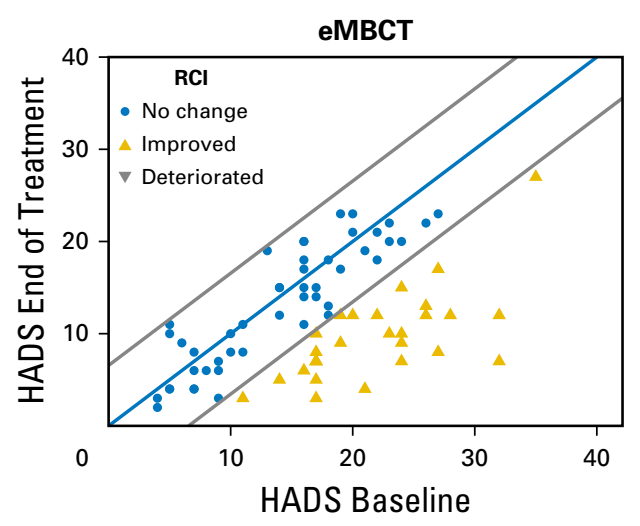

C

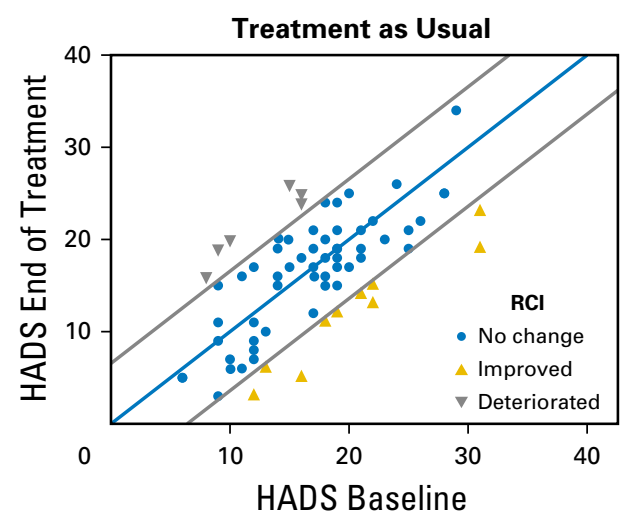

Fig 2. Change in Hospital Anxiety and Depression Scale (HADS) scores between baseline and postintervention for (A) mindfulness-based cognitive therapy (MBCT), (B) Internet-based mindful-based cognitive therapy (eMBCT), and (C) treatment as usual (TAU) groups. The blue diagonal line represents no change in HADS between baseline and postintervention, and the gray upper and lower lines represent the upper (above indicates deteriorated) and lower (below indicates improved) bounds of the $95 \% \mathrm{Cl}$ of the Jacobson-Truax reliable change index (RCI). types of cancer. Because one inclusion criterion was the ability and willingness to attend both MBCT and eMBCT, the sampling frame for the current study was probably not representative of patients who would prefer eMBCT in clinical practice. Because treatment preference is often positively correlated with treatment outcome, ${ }^{45}$ we would expect that this RCT underestimated rather than overestimated the effects of eMBCT.

In terms of research implications, long-term results should be gathered to examine the stability of effects. In addition, data on cost-utility of MBIs in patients with cancer should be collected. ${ }^{46}$ Internet interventions do not involve the costs of transportation, traveling time, space, equipment, cleaning, and other overhead expenses and thus could be more cost effective. Possible mediators of the effect, such as mindfulness skills or rumination, should be further investigated. ${ }^{47,48}$ Moreover, mediation analyses could also examine possible differences in adherence in both MBCT and eMBCT.

In terms of clinical implications, implementation of eMBCT could make MBIs more accessible for patients with cancer without having to compromise intervention efficacy. However, intervention dropout could possibly be improved by the delivery mode of eMBIs. ${ }^{49}$ Qualitative work demonstrates that aspects such as the individual nature and the asynchronous interaction of the eMBCT used in this study are helpful for some patients. ${ }^{50}$ Future studies should assess how different eMBCT designs (eg, blended designs

Table 4. Clinically Significant Improvement Measured by Jacobson-Truax Reliable Change Index on HADS and Psychiatric Diagnosis Between Baseline and Postintervention for MBCT, eMBCT, and TAU Groups

\begin{tabular}{|c|c|c|c|c|c|c|c|c|c|c|c|c|c|c|c|c|}
\hline \multirow[b]{3}{*}{ Improvement } & \multicolumn{8}{|c|}{ Reliable Change Index $(n=209)$} & \multicolumn{8}{|c|}{ Psychiatric Diagnosis $(n=202)$} \\
\hline & \multicolumn{2}{|c|}{$\begin{array}{c}\text { MBCT } \\
(n=64)\end{array}$} & \multirow[b]{2}{*}{$P^{*}$} & \multicolumn{2}{|c|}{$\begin{array}{l}\text { eMBCT } \\
(\mathrm{n}=75)\end{array}$} & \multirow[b]{2}{*}{$P^{*}$} & \multicolumn{2}{|c|}{$\begin{array}{c}\text { TAU } \\
(n=70)\end{array}$} & \multicolumn{2}{|c|}{$\begin{array}{c}\text { MBCT } \\
(n=64)\end{array}$} & \multirow[b]{2}{*}{$P^{*}$} & \multicolumn{2}{|c|}{$\begin{array}{c}\text { eMBCT } \\
(n=76)\end{array}$} & \multirow[b]{2}{*}{$P^{*}$} & \multicolumn{2}{|c|}{$\begin{array}{c}\text { TAU } \\
(\mathrm{n}=62)\end{array}$} \\
\hline & No. & $\%$ & & No. & $\%$ & & No. & $\%$ & No. & $\%$ & & No. & $\%$ & & No. & $\%$ \\
\hline Improved & 23 & 36 & .004 & 28 & 37 & .002 & 10 & 14 & 21 & 33 & .030 & 22 & 29 & .076 & 10 & 16 \\
\hline No change & 39 & 61 & & 47 & 63 & & 54 & 77 & 34 & 67 & & 50 & 66 & & 49 & 79 \\
\hline Deteriorated & 2 & 3 & .184 & 0 & 0 & .010 & 6 & 9 & 0 & 0 & .075 & 4 & 5 & .910 & 3 & 5 \\
\hline
\end{tabular}

Abbreviations: eMBCT, Internet-based MBCT; HADS, Hospital Anxiety and Depression Scale; MBCT, mindfulness-based cognitive therapy; TAU, treatment as usual. *Between group $\chi^{2}$ tests for the respective condition compared with TAU. 
combining the advantages of face-to-face and Internet-based elements) $)^{51}$ could further improve intervention accessibility, adherence, and effectiveness. ${ }^{50}$

\section{AUTHORS' DISCLOSURES OF POTENTIAL CONFLICTS OF INTEREST}

Disclosures provided by the authors are available with this article at jco.org.
AUTHOR CONTRIBUTIONS

Conception and design: Linda Carlson, Marije van der Lee, Anne Speckens

Collection and assembly of data: Félix Compen, Else Bisseling

Data analysis and interpretation: Félix Compen, Else Bisseling, Melanie

Schellekens, Rogier Donders, Marije van der Lee, Anne Speckens

Manuscript writing: All authors

Final approval of manuscript: All authors

Accountable for all aspects of the work: All authors

\section{REFERENCES}

1. Bray F: Transitions in human development and the global cancer burden, in Wild CP, Stewart B (eds): World Cancer Report 2014. Lyon, France, International Agency for Research on Cancer, 2014, pp 54-68

2. Carlson LE, Angen $M$, Cullum J, et al: High levels of untreated distress and fatigue in cancer patients. Br J Cancer 90:2297-2304, 2004

3. Mitchell AJ, Chan M, Bhatti $H$, et al: Prevalence of depression, anxiety, and adjustment disorder in oncological, haematological, and palliative-care settings: A meta-analysis of 94 interview-based studies. Lancet Oncol 12:160-174, 2011

4. Mitchell AJ, Ferguson DW, Gill, J., et al: Depression and anxiety in long-term cancer survivors compared with spouses and healthy controls: A systematic review and meta-analysis. Lancet Oncol 14:721-732, 2013

5. Kabat-Zinn J: Full catastrophe living (revised edition): Using the Wisdom of Your Body and Mind to Face Stress, Pain and Illness using mindfulness meditation. Piatkus, 2013

6. Segal ZV, Williams JMG, Teasdale JD: Mindfulness-based cognitive therapy for depression: A new approach to relapse prevention. New York, NY Guilford Press, 2013

7. Piet J, Würtzen $H$, Zachariae R: The effect of mindfulness-based therapy on symptoms of anxiety and depression in adult cancer patients and survivors: A systematic review and meta-analysis. J Consult Clin Psychol 80:1007-1020, 2012

8. Carlson LE, Doll R, Stephen J, et al: Randomized controlled trial of mindfulness-based cancer recovery versus supportive expressive group therapy for distressed survivors of breast cancer. J Clin Oncol 31:3119-3126, 2013

9. Garland SN, Carlson LE, Stephens AJ, et al: Mindfulness-based stress reduction compared with cognitive behavioral therapy for the treatment of insomnia comorbid with cancer: A randomized, partially blinded, noninferiority trial. J Clin Oncol 32: 449-457, 2014

10. Lengacher $C A$, Reich $R R$, Paterson $C L$, et al: Examination of broad symptom improvement resulting from mindfulness-based stress reduction in breast cancer survivors: A randomized controlled trial. J Clin Oncol 34:2827-2834, 2016

11. Johannsen $M, O^{\prime}$ Connor M, O'Toole MS, et al: Efficacy of mindfulness-based cognitive therapy on late post-treatment pain in women treated for primary breast cancer: A randomized controlled trial. $J$ Clin Oncol 34:3390-3399, 2016

12. Schellekens MPJ, van den Hurk DGM, Prins $J B$, et al: Mindfulness-based stress reduction added to care as usual for lung cancer patients and/or their partners: A multicentre randomized controlled trial. Psychooncology 26:2118-2126, 2017

13. Carlson LE, Tamagawa $R$, Stephen J, et al: Randomized-controlled trial of mindfulness-based cancer recovery versus supportive expressive group therapy among distressed breast cancer survivors (MINDSET): Long-term follow-up results. Psychooncology 25:750-759, 2016

14. Zernicke KA, Campbell TS, Speca M, et al: A randomized wait-list controlled trial of feasibility and efficacy of an online mindfulness-based cancer recovery program: The eTherapy for cancer applying mindfulness trial. Psychosom Med 76:257-267, 2014

15. Brebach R, Sharpe L, Costa DSJ, et al: Psychological intervention targeting distress for cancer patients: A meta-analytic study investigating uptake and adherence. Psychooncology 25:882-890, 2016

16. Spijkerman MPJ, Pots WTM, Bohlmeijer ET: Effectiveness of online mindfulness-based interventions in improving mental health: A review and meta-analysis of randomised controlled trials. Clin Psychol Rev 45:102-114, 2016

17. Andersson G: Internet-delivered psychological treatments. Annu Rev Clin Psychol 12:157-179, 2016

18. Zernicke KA, Campbell TS, Speca M, et al: The eCALM Trial: eTherapy for cancer applying mindfulness: Online mindfulness-based cancer recovery program for underserved individuals living with cancer in Alberta: Protocol development for a randomized wait-list controlled clinical trial. BMC Complement Altern Med 13:34, 2016

19. Everts FZB, van der Lee ML, de Jager Meezenbroek $E$ : Web-based individual mindfulnessbased cognitive therapy for cancer-related fatiguea pilot study. Internet Interventions 2:200-213, 2015

20. Schulz KF, Altman DG, Moher D: CONSORT 2010 statement: Updated guidelines for reporting parallel group randomised trials. BMC Med 8:18, 2010

21. Compen FR, Bisseling EM, Van der Lee ML, et al: Study protocol of a multicenter randomized controlled trial comparing the effectiveness of group and individual Internet-based mindfulness-based cognitive therapy with treatment as usual in reducing psychological distress in cancer patients: The BeMind study. BMC Psychol 3:27, 2015

22. Crane $C$, Williams JMG: Factors associated with attrition from mindfulness-based cognitive therapy in patients with a history of suicidal depression. Mindfulness (N Y) 1:10-20, 2010

23. Hakkaart-van Roijen $L$, Straten A, Donker M, et al: Trimbos/iMTA Questionnaire for Costs Associated With Psychiatric Illness (TiC-P). Rotterdam, the Netherlands, Institute for Medical Technology Assessment, 2002

24. UK Network of Mindfulness Teacher Training Organisations: UK Network for Mindfulness-Based Teachers: Good practice guidelines for teaching mindfulness based courses, 2015. https://www. mindfulnessteachersuk.org.uk/pdf/UK\% $20 \mathrm{MB}$ \% 20teacher\%20GPG\%202015\%20final\%202.pdf

25. Crane RS, Kuyken W, Williams JM, et al: Competence in teaching mindfulness-based courses: Concepts, development and assessment. Mindfulness (N Y) 3:76-84, 2012

26. Zigmond AS, Snaith RP: The Hospital Anxiety And Depression Scale. Acta Psychiatr Scand 67: 361-370, 1983

27. Spinhoven P, Ormel J, Sloekers PP, et al: A validation study of the Hospital Anxiety and Depression Scale (HADS) in different groups of Dutch subjects. Psychol Med 27:363-370, 1997

28. Akechi $T$, Okuyama $T$, Sugawara $Y$, et al: Screening for depression in terminally ill cancer patients in Japan. J Pain Symptom Manage 31:5-12, 2006

29. First MB, Spitzer RL, Gibbon M, et al. Structured Clinical Interview for DSM-IV® Axis I Disorders (SCID-I). Clinician Version, Administration Booklet. Washington, DC, American Psychiatric Publishing, 2012

30. Simard S, Savard J: Fear of Cancer Recurrence Inventory: Development and initial validation of a multidimensional measure of fear of cancer recurrence. Support Care Cancer 17:241-251, 2009

31. van Helmondt SJ, van der Lee ML, de Vries J: Translation and validation of the Dutch version of the Fear of Cancer Recurrence Inventory (FCRI-NL). J Psychosom Res 102:21-28, 2017

32. Trapnell PD, Campbell JD: Private selfconsciousness and the five-factor model of personality: Distinguishing rumination from reflection. J Pers Soc Psychol 76:284-304, 1999

33. Ware J Jr, Kosinski M, Keller SD: A 12-item Short-Form health survey: Construction of scales and preliminary tests of reliability and validity. Med Care 34:220-233, 1996

34. Mols F, Pelle AJ, Kupper N: Normative data of the SF-12 health survey with validation using postmyocardial infarction patients in the Dutch population. Qual Life Res 18:403-414, 2009

35. Bohlmeijer $E$, ten Klooster PM, Fledderus $M$ et al: Psychometric properties of the Five Facet Mindfulness Questionnaire in depressed adults and development of a short form. Assessment 18: 308-320, 2011

36. Keyes CL: Mental illness and/or mental health? Investigating axioms of the complete state model of health. J Consult Clin Psychol 73:539-548, 2005

37. Costa PT, McCrae RR: Revised NEO Personality Inventory (NEO-PI-R) and the Five Factor Inventory (NEO-FFI): Professional Manual. Odessa, FL, Psychological Assessment Resources, 1992

38. McGough JJ, Faraone SV: Estimating the size of treatment effects: Moving beyond $\mathrm{p}$ values. Psychiatry (Edgmont Pa) 6:21-29, 2009 
39. Cohen J: Statistical Power Analysis for the Behavioral Sciences (revised ed). New York, NY, Academic Press, 1977

40. Jacobson NS, Truax P: Clinical significance: $A$ statistical approach to defining meaningful change in psychotherapy research. J Consult Clin Psychol 59: 12-19, 1991

41. Schellekens MP, Jansen ET, Willemse HH, et al: A qualitative study on mindfulness-based stress reduction for breast cancer patients: How women experience participating with fellow patients. Support Care Cancer 24:1813-1820, 2016

42. Melville KM, Casey LM, Kavanagh DJ: Dropout from Internet-based treatment for psychological disorders. Br J Clin Psychol 49:455-471, 2010

43. van Scheppingen C, Schroevers MJ, Pool G, et al: Is implementing screening for distress an efficient means to recruit patients to a psychological intervention trial? Psychooncology 23:516-523, 2014

44. Garssen B, Van der Lee M, Van der Poll A, et al: Characteristics of patients in routine psychooncological care, and changes in outcome variables during and after their treatment. Psychol Health 31: 1237-1254, 2016

45. Swift JK, Callahan JL: The impact of client treatment preferences on outcome: A meta-analysis. J Clin Psychol 65:368-381, 2009

46. Carlson LE, Bultz BD: Efficacy and medical cost offset of psychosocial interventions in cancer care: Making the case for economic analyses. Psychooncology 13:837-849, 2004; discussion 850-856

47. Kazdin AE: Mediators and mechanisms of change in psychotherapy research. Annu Rev Clin Psychol 3:1-27, 2007
48. Gu J, Strauss $C$, Bond $R$, et al: How do mindfulness-based cognitive therapy and mindfulnessbased stress reduction improve mental health and wellbeing? A systematic review and meta-analysis of mediation studies. Clin Psychol Rev 37:1-12, 2015

49. Toivonen KI, Zernicke K, Carlson LE: Webbased mindfulness interventions for people with physical health conditions: Systematic review. J Med Internet Res 19:e303, 2017

50. Compen FR, Bisseling EM, Schellekens MP, et al: Mindfulness-based cognitive therapy for cancer patients delivered via Internet: Qualitative study of patient and therapist barriers and facilitators. J Med Internet Res 19:e407, 2017

51. Wentzel J, van der Vaart R, Bohlmeijer ET, et al: Mixing online and face-to-face therapy: How to benefit from blended care in mental health care. JMIR Ment Health 3:e9, 2016

\section{Affiliations}

Félix Compen, Else Bisseling, Melanie Schellekens, Rogier Donders, and Anne Speckens, Radboud University Nijmegen, Nijmegen; Félix Compen, Else Bisseling, and Marije van der Lee, Helen Dowling Institute, Bilthoven, the Netherlands; Linda Carlson, University of Calgary, Calgary, Canada.

\section{Support}

Supported by Pink Ribbon (2012.WO14.C153). L.C. holds the Enbridge Research Chair in Psychosocial Oncology, co-funded by the Canadian Cancer Society Albert/Northwest Territories Division and the Alberta Cancer Foundation.

\section{Prior Presentation}

Presented at the 14th International Congress of Behavioral Medicine, Melbourne, Australia, December 7-9, 2016; and the 19th World Congress of International Psycho-Oncology and Psychosocial Academy, Berlin, Germany, August 14-18, 2017. 
AUTHORS' DISCLOSURES OF POTENTIAL CONFLICTS OF INTEREST

Face-to-Face and Internet-Based Mindfulness-Based Cognitive Therapy Compared With Treatment as Usual in Reducing Psychological Distress in Patients With Cancer: A Multicenter Randomized Controlled Trial

The following represents disclosure information provided by authors of this manuscript. All relationships are considered compensated. Relationships are self-held unless noted. I = Immediate Family Member, Inst = My Institution. Relationships may not relate to the subject matter of this manuscript. For more information about ASCO's conflict of interest policy, please refer to www.asco.org/rwc or ascopubs.org/jco/site/ifc.

\section{Félix Compen}

No relationship to disclose

Else Bisseling

No relationship to disclose

\section{Melanie Schellekens}

No relationship to disclose

\section{Rogier Donders}

No relationship to disclose

\section{Linda Carlson}

No relationship to disclose

\section{Marije van der Lee}

No relationship to disclose

\section{Anne Speckens}

No relationship to disclose 


\section{Acknowledgment}

We are grateful to the patients for their participation in this trial and to physicians and therapists from Radboud University Medical Centre, Nijmegen; Helen Dowling Institute, Bilthoven; De Vruchtenburg, Leiden/Rotterdam; Ingeborg Douwes Centrum, Amsterdam; Het Behouden Huys, Haren; and Jeroen Bosch Ziekenhuis, Den Bosch, the Netherlands, for referring patients to the trial. We would like to thank Heidi Willemse, Eva Witteveen, Merel Brands, and David Huijts for their help in data collection; and Ellen Jansen, Rinie van den Boogaart, Coen Völker, Joyce Vermeer, Bert Vedder, Ineke Vogel, Mira Reus, Lot Heijke, Maria van Balen, Annetje Brunner, Maya Schroevers, Stephanie Verhoeven, and Janine Mutsaers for providing the online and group mindfulness-based cognitive therapy. We thank Caroline Hoffman for her contribution to the supervision of the therapists.

\section{Appendix}

Table A1. Adverse Events Reported per Condition

\begin{tabular}{|c|c|c|c|}
\hline Participant & MBCT $(n=9)$ & eMBCT $(n=6)$ & $\mathrm{TAU}(\mathrm{n}=9)$ \\
\hline Intervention completer & $\begin{array}{l}\text { Pneumonia }(n=1) \\
\text { Cancer recurrence }(n=1) \\
\text { Questionnaires too burdensome }(n=4)\end{array}$ & $\begin{array}{l}\text { Questionnaires too burdensome }(n=2) \\
\text { Needed additional psychological help }(n=1)\end{array}$ & $\begin{array}{l}\text { Illness progression }(n=3) \\
\text { Questionnaires too burdensome }(n=3) \\
\text { Needed additional psychological help }(n=2) \\
\text { Reported high distress }(n=1)\end{array}$ \\
\hline Intervention dropout & $\begin{array}{l}\text { Surgery }(n=1) \\
\text { Deceased }(n=1) \\
\text { Unspecified medical reason }(n=1)\end{array}$ & $\begin{array}{l}\text { Illness progression }(n=1) \\
\text { Unspecified medical reason }(n=2)\end{array}$ & \\
\hline
\end{tabular}

Abbreviations: eMBCT, Internet-based MBCT; MBCT, mindfulness-based cognitive therapy; TAU, treatment as usual. 